\title{
A new freshwater basal eucryptodiran turtle from the Lower Cretaceous of Spain
}

Adán Pérez-García, Marcelo S. de la Fuente, and Francisco Ortega

Acta Palaeontologica Polonica 57 (2), 2012: 285-298 doi: http://dx.doi.org/10.4202/app.2011.0031

A freshwater turtle from the lithographic limestone of Las Hoyas (Barremian of Cuenca, Spain) is described as a new genus and species of Eucryptodira, Hoyasemys jimenezi. The holotype consists of the skull, lower jaw, carapace, plastron, vertebral column, pectoral and pelvic girdle remains, and fore- and hindlimbs. Hoyasemys jimenezi gen. et sp. nov. is characterized by three pairs of blind oblique depressions on the ventral surface of the basisphenoid, and a character combination composed, among others, of the articulation between the fourth and fifth cervical vertebrae through a cotyle in the fourth and a condyle in the fifth, amphicoelous caudal centra, and most digits of manus and pes with three elongated phalanges. This study allows clarification of the systematic position of a species of uncertain affinity often identified as "chelydroid" in appearance. A phylogenetic analysis shows Hoyasemys jimenezi gen. et sp. nov. forms a monophyletic group with Judithemys sukhanovi, Dracochelys bicuspis, Sinemys lens, and Ordosemys leios, collectively the sister groupof crown Cryptodira.

Key words: Testudines, Eucryptodira, freshwater turtle, Barremian, Cretaceous, Las Hoyas, Spain.

Adán Pérez-García [paleontologo@gmail.com], Departamento de Paleontología, Facultad de Ciencias Geológicas, Universidad Complutense de Madrid, José Antonio Novais 2, 28040 Ciudad Universitaria, Madrid, Spain; Marcelo S. de la Fuente [mdelafu@gmail.com], Departamento de Paleontología, Museo de Historia Natural de San Rafael, Parque Mariano Moreno S/No (5600), San Rafael, Provincia de Mendoza, Argentina; Francisco Ortega [fortega@ccia.uned.es], Grupo de Biología, Facultad de Ciencias, UNED, Senda del Rey 9, 28040 Madrid, Spain. 
distribution, and reproduction in any medium, provided the original author and source are credited.

Faf Full text $(684.0 \mathrm{kB})$ 